\title{
Assessing Dementia in Resource-Poor Regions
}

\author{
Gladys E. Maestre
}

Published online: 5 August 2012

(C) The Author(s) 2012. This article is published with open access at Springerlink.com

\begin{abstract}
The numbers and proportions of elderly are increasing rapidly in developing countries, where prevalence of dementia is often high. Providing cost-effective services for dementia sufferers and their caregivers in these resourcepoor regions poses numerous challenges; developing resources for diagnosis must be the first step. Capacity building for diagnosis involves training and education of healthcare providers, as well as the general public, development of infrastructure, and resolution of economic and ethical issues. Recent progress in some low-to-middle-income countries (LMICs) provides evidence that partnerships between wealthy and resource-poor countries, and between developing countries, can improve diagnostic capabilities. Without the involvement of the mental health community of developed countries in such capacity-building programs, dementia in the developing world is a disaster waiting to happen.
\end{abstract}

Keywords Developing countries $\cdot$ Low-to-middle-income countries · Resource-poor · Population demography ·

Elderly $\cdot$ Dementia $\cdot$ Prevalence $\cdot$ Diagnosis $\cdot$ Capacity building $\cdot$ Challenges $\cdot$ Obstacles $\cdot$ International partnerships $\cdot$ Cost-benefit analysis $\cdot$ Economics $\cdot$ Ethics

\section{G. E. Maestre $(\triangle)$}

Laboratory of Neurosciences, University of Zulia,

Edificio del Instituto de Enfermedades Cardiovasculares de la

Universidad del Zulia, Primer Piso,

Av Universidad diagonal al MACZUL,

Maracaibo 4002 Zulia, Venezuela

e-mail: gem6@columbia.edu

\section{G. E. Maestre}

Departments of Psychiatry and Neurology, and Gertrude H. Sergievsky Center, Columbia University, PH 19 Room 315, 622 West 168th Street, New York, NY 10032, USA

\section{Introduction}

Dementia disrupts normal functioning of affected individuals and their families, imposing significant social and economic burdens. The problem is especially severe in low-tomiddle-income countries (LMICs), where dementia is the most important independent contributor to disability in the elderly [1] and resources to diagnose and treat dementia are limited. By the mid-21st century, $78 \%$ of the world's elderly population will reside in LMICs, with expected concomitant increases in dementia cases [2]. Prevalence of dementia is relatively low in some LMICs, but other regions have a prevalence similar to or higher than that in developed countries [3]. For example, Latin America and the Caribbean (LAC) have the world's highest prevalence of dementia (8.5\% in subjects $\geq 60$ years vs. $6.4 \%$ in the U.S.) [3-5].

It is in resource-poor regions with a high prevalence of dementia that cost-effective health care targeted at vulnerable populations is most needed. However, dementia care is problematic, even in high-income countries, many patients with dementia remain undiagnosed and, therefore, untreated $[6,7 \cdot]$. The governments of LMICs and international healthcare agencies have tended to disregard chronic conditions of the elderly, due to more immediate and widespread problems of malnutrition and communicable diseases. However, the World Health Organization (WHO) recently released guidelines for increasing treatment of dementia in LMICs [8] and urged governments to consider dementia as a public health priority $[9 \bullet \bullet]$. Developing effective approaches for diagnosis is the first step toward providing adequate services for dementia sufferers and their caregivers. In this article, I review strategies used to diagnose dementia in resourcepoor regions, discuss the particular challenges associated with those assessments, describe some recent strategies for developing and improving diagnosis in poor countries, and consider how diagnosis of dementia might be improved in the future. 


\section{Prevalence of Dementia in the Developing World}

There are many estimates of age-specific or age-and-genderspecific dementia prevalence in the developed world, based on epidemiological studies. However, WHO identified only 64 such studies in 14 developing countries [9••]. Where such data are not available, estimates have been derived from a Delphi consensus of experts [10]. Furthermore, the age-specific and age-and-gender-specific estimates for developing countries are often compared with estimates for developed countries, despite different population structures. Therefore, estimates of the prevalence of dementia in the developing world should be viewed with caution.

Most estimates of the prevalence of dementia in people older than 60 years fall between $5 \%$ and $7 \%$ [3]. Variation in actual prevalence - as opposed to variation in detected dementia - is influenced by a number of factors. For example, low prevalence in sub-Saharan Africa (2.1\%) might be due to selective mortality of people younger than 60 years [11]. High prevalence in LAC (8.5\%) might be related to the high occurrence or confluence of genetic and/or environmental risk factors [5]. For example, increased mobility of individuals and families has spread genetic isolates with an extremely high prevalence of dementia, such as those with early-onset Alzheimer's disease in Medellin, Colombia [12] and Huntington's disease in Maracaibo, Venezuela [13]. The fact that expression of the disease occurs after reproductive age might also contribute to its spread. Finally, in consanguineous marriages, which are relatively common in some resource-poor areas, dementia in either or both parents increases the risk of having children who will be affected in the future [14].

Population demography and other factors play an important role in determining the prevalence of dementia. Both prevalence and incidence of dementia increase exponentially with age, making age the main risk factor for the disease $[9 \bullet, 15]$. The number of people at risk of dementia in the developing world will increase rapidly during the next 50 years, because the population and proportion will grow faster for the elderly ( $\geq 60$ years) than for other age groups [16]. In India, for example, the population of individuals $\geq 80$ years will increase from 5.4 million in 2001 to 32 million in 2051 [17]. In addition to aging populations, gradual improvements in health care in developing countries are probably increasing the time that affected individuals survive with dementia [18]. Other factors that might affect the incidence of certain types of dementia - for example, cardiovascular risk factors, such as obesity, metabolic syndrome, and sedentary life - are also escalating much more rapidly in developing than in developed countries [19-22]. In consequence, the dementia burden in the developing world is expected to increase significantly.

\section{Diagnosing Dementia}

Diagnosis of dementia and accurate determination of subtype early in the course of the disease are crucial for optimal clinical care and management. Traditionally, dementia has been defined as a global impairment of intellectual function that requires anatomopathological examination to provide accurate diagnosis and differentiation among subtypes [23-25]. However, research involving biomarkers and neurogenetics now allows identification of patients years, perhaps decades, before the diagnosis of dementia is made, such as in presymptomatic Alzheimer's disease [26].

The National Institute on Aging and the Alzheimer's Association recently released revised criteria for all-cause dementia [27], which include neuropsychiatric or cognitive symptoms, rather than just cognitive deficits, that interfere with daily functioning, represent a decline from previous levels, and are not explained by delirium or major psychiatric disorders. A new category was added, primarily intended for research purposes: dementia due to possible or probable Alzheimer's disease with evidence of the Alzheimer pathology. The new category requires that a subject meeting the clinical criteria for Alzheimer's disease also exhibits changes in biomarkers related to the current understanding of the pathophysiology [28]. The revised criteria for Alzheimer's disease include a predementia stage, in which a subject is asymptomatic but shows evidence of the underlying brain pathology specific for Alzheimer's disease. These new criteria will be of limited use in the developing world, because the ability to detect relevant biomarkers and pathologies is low, due to the high cost of testing and the lack of infrastructure.

To establish a diagnosis of dementia, an evaluation focuses on, as a clinical syndrome, cognitive performance and behavior of the subject, changes in the activities of daily living, and exclusion of other possible causes. Diagnosing dementia, particularly in the early stages, is neither simple nor straightforward. The clinical interview is considered to be an art. The first step is usually to elicit a complete history of signs and symptoms, recalled and recounted by the patient and/or an informant. A highly skilled interviewer structures the interaction as a conversation, establishing rapport and confidence and enabling the patient to fully disclose even embarrassing details. Neuropsychological, neuroimaging, and other diagnostic tests are used to distinguish pathological changes within the brain that characterize different dementia syndromes. Interpretation of the clinical interview and test results requires training and often varies among individuals making the judgment.

The clinician uses as many tools as possible-clinical examination, laboratory tests, neuroimaging, genetic testing - to determine the underlying pathology or type of dementia and establish the best therapeutic course as 
early as possible. However, the diagnostic profiles of different dementia subtypes are not $100 \%$ distinct, and the correlation between clinical and pathological diagnoses is not perfect, even when based on the most advanced tools [18]. Furthermore, diagnostic profiles exhibit a great deal of heterogeneity, even among patients who share the same pathology, and some are frankly atypical [29]. Because of these challenges, accurate diagnosis requires an experienced clinician, supported by an array of complex tools $[7 \cdot, 30]$

\section{Obstacles to Diagnosis of Dementia in Poor Populations}

Many factors contribute to limited diagnosis of dementia in resource-poor areas (Table 1). Three major obstacles are (1) low health literacy, (2) limited access to health care, and (3) the stigma associated with dementia. The extent of health illiteracy, including ignorance of the connection between behavioral, cognitive, or physical signs and the disease [31], is difficult to estimate but very important, as is lack of awareness that appropriate care/treatment is available [32]. Surprisingly, such ignorance often exists in educated individuals and even in healthcare professionals [33]. Lack of knowledge in healthcare workers particularly affects their ability to recognize early signs of dementia. Many people in developing countries believe that the characteristic signs of dementia are part of normal aging or a nonpathological deviation [34]. Improving health literacy is the goal of health promotion and education programs, which are often given low priority in resource-poor settings. On the other hand, the impact of a lack of specific or scientific knowledge should not be overestimated. Cultures in developing

Table 1 Likely causes of limited dementia diagnoses in resource-poor areas

- Initial symptoms are subtle and fluctuating

- Inability to recognize cognitive, behavioral, or functional impairment as a consequence of dementing illness

- Accommodation, denial, and rationalization of symptoms as due to aging and adverse circumstances

- High tolerance for health problems in elderly individuals

- Person with early symptoms does not feel the need to seek treatment

- Relatives do not believe symptoms are treatable

- Demoralization regarding medical assistance, due to ignorance of available treatments

- Do not know where to go for diagnosis

- Family is afraid of psychiatric stigma (demented is often equated to being crazy)

- Physician underemphasizes the importance of symptoms

- Perception that physicians will miss the diagnosis and will make the family waste time and money in apparently unnecessary tests

- Costs of diagnosis in terms of effort, time, economic costs countries often have knowledge about signs, based on common sense and traditional practices, that informs choices about care/treatment [35].

Limited access to health care is a universal problem in diagnosing dementia in LMICs. Geriatric care is generally minimal, and there is virtually no concept of continuing care for aging adults [36-38]. In addition to inadequate numbers of personnel and facilities, the costs involved in accessing services and long waits for appointments are common barriers. If an elderly person has to travel a long distance to receive a diagnosis and treatment, he or she must weigh the likelihood of improvement against the difficulties and costs of travel [39]. Travel where public transportation is not adequate for people with physical disabilities can be a limitation. Additionally, the patient and his or her caretakers might fear that there is no effective treatment, that treatment will require expensive drugs, or that the patient might have to stay at an asylum or hospice. Any or all of these factors might prevent a potential patient or caregiver from seeking medical attention, particularly in the case of a chronic condition that has established slowly, such as dementia.

The social stigma associated with mental health problems is a barrier to diagnosing dementia [40-42]. When dementia is medicalized and acquires the label of a disease, the social value of the individual and his or her family often suffers $[11,35]$. Even when it is not labeled as a disease, signs of dementia are a fairly common condition in many resourcepoor regions, and most people have a negative expectation of recovery [11]. Behavioral changes associated with dementia sometimes elicit abuse, neglect, or loss of family members' respect [40]. Even healthcare workers are sometimes guilty of a negative attitude toward dementia patients [41-43].

In addition to health illiteracy, poor access to healthcare, and social stigma, diagnosing dementia in poor populations remains problematic for a number of reasons. Although the clinical features and complications of dementia have been relatively well defined [44], expression of behavioral and cognitive signs and symptoms might be modulated by socioeconomic conditions [41], possibly accounting for some of the phenotypic heterogeneity of various subtypes. Longterm studies are needed of the effects of lifestyles in developing countries on each of the dementia subtypes [45]. Although some genetic and environmental modifiers have been identified [46-48], it is still not possible to predict the likely course of the illness, even when dominant mutations are present [45]. The effects of concomitant comorbidities, such as hypertension and diabetes, are not clearly understood, particularly when untreated or uncontrolled for most of the life of the patient [49].

Practical approaches to overcoming some obstacles to diagnosis of dementia have been addressed in the context 
of minority populations [50•] (Table 2). There are other obstacles, particularly to early diagnosis of dementia, that are beyond the scope of this article [51]. However, it is important to recognize that economic crises, epidemics, famine, war, displacement, and natural disasters have devastating effects on populations in resource-poor countries, which relegate the health of elderly individuals to low priority $[52,53]$. Barriers to improvement of dementia diagnosis and care are similar to those confronting mental health services [54]. Overcoming those barriers will require changes in politics, leadership, planning, advocacy, and participation [55].

Inarguably, it is crucial to reliably diagnose individuals with dementia in order to carry out clinical trials for treatments, to advance genetic studies, and to proceed with epidemiological studies that allow the establishment of specific risk factors. However, because there is no definite marker for the disease (except when relatively rare dominant genetic alterations are present and consistent with symptomatology), population screening and preclinical diagnosis in general populations are not yet cost effective and have not been implemented even in wealthy countries. Even screening for the ApoE-4 allele, the strongest risk factor for Alzheimer's disease in most populations, is not generally recommended because of prognostic uncertainty [56]. In contrast, screening populations at high risk for dementia (e.g., elderly individuals with a medical history of risk factors for dementia) might be justifiable, but systematic recommendations have not been developed [57]. Nevertheless, the benefits of early diagnosis, coupled with proper care and caregiver support, outweigh the costs of diagnosis in developing countries [7•]. Although the only specific treatment for dementia, cholinesterase inhibitors and/or memantine, is available to a relatively small number of patients, primarily in wealthy countries [58], therapeutic interventions are currently being tested. Now is the time to prepare for their availability.

\section{Human Resource Capacity for Diagnosing Dementia in LMICs}

While the number of specialty centers for dementia care has grown considerably in wealthier countries, apart from a few evolving areas [59-61], facilities for diagnosis and treatment of dementia are scarce in LMICs [62].

There is also a paucity of specialists devoted to health care of the elderly and to brain and mental health disorders [63-68]. Neuropsychologists and biometricians who are competent to administer standardized tests to local populations and interpret the results are rare, so normative values that can be appropriately applied by clinicians in diagnosing dementia are even rarer $[51,69]$. The Kyoto Declaration of Alzheimer's Disease International (ADI), on the basis of the recommendations of WHO 2001 [70], recommended integrating dementia outreach into existing primary health care, and WHO developed evidence-based guidelines for management of dementia by nonspecialists in LMICs [8]. Nevertheless, most primary care physicians and even many specialists in developing countries do not receive suitable training to diagnose dementia and its subtypes [40], despite efforts to remedy this situation during the past few years [71].

Building capacity to recognize and accurately diagnose dementia in LMICs, by training local healthcare personnel, is necessary to expand access to and improve mental health care $[7 \cdot, 64]$. To date, most initiatives for training healthcare workers in dementia diagnosis have depended largely on face-to-face interactions [72, 73], but the role of distance and Web- or mobile-telephone-based training is increasing [74-76]. However, none of the Web-based programs appear to offer training in different languages or to include specific information on assessment of individuals with low levels of education. Capacity-building efforts should also aim to improve referral pathways and promote multidisciplinary care of dementia patients, which will require training of nurses, social workers, and other healthcare personnel,
Table 2 Options for and challenges to confronting critical issues in the diagnosis of dementia among minorities that are applicable to resource-poor regions

\begin{tabular}{lll}
\hline Issues & Options & Challenges \\
\hline $\begin{array}{l}\text { Need culture-unbiased } \\
\text { instruments }\end{array}$ & $\begin{array}{l}\text { Adapt existing tests } \\
\text { Develop new instruments }\end{array}$ & $\begin{array}{l}\text { Comparability } \\
\text { Alteration of psychometric properties } \\
\text { Need local normative values }\end{array}$ \\
$\begin{array}{l}\text { Undetermined value of } \\
\text { informant data }\end{array}$ & Identify key informant & $\begin{array}{l}\text { Stigma } \\
\text { Oversimplification of complex social } \\
\text { relationships and environments }\end{array}$ \\
$\begin{array}{l}\text { Assessment of daily } \\
\text { functioning }\end{array}$ & Home-based interview & $\begin{array}{l}\text { Time consuming } \\
\text { Interpretation of conflicting } \\
\text { information }\end{array}$ \\
& Skilled clinician & Specific to setting \\
& Multidisciplinary & Experienced clinical judgment \\
& consensus on diagnosis & Intimate knowledge of culture \\
\hline
\end{tabular}


in addition to specialists, such as geriatricians, neurologists, and psychiatrists.

Continued medical education in much of the developing world is currently subsidized by pharmaceutical industries [77], without strict regulations on content or selection of presenters. It is not surprising that many educational and dissemination programs on dementia are biased toward pharmacological approaches and downplay nonpharmacological strategies for managing behavioral symptoms [78]. Improvement and expansion of those programs to include dementia require input by both large international agencies and academia. Little is likely to be accomplished without their recognition of dementia as a public health problem in poor populations, with emphasis on prevention of risk factors, early diagnosis, and improved caregiving.

\section{Economic Issues Affecting Diagnosis of Dementia in LMIC}

Economic issues are critical to the control and management of dementia in LMICs [79]. Economic issues also drive the perceived need for early diagnosis of dementia and differentiation of subtypes [7•]. To develop appropriate and effective policies, resource-poor countries require information about the overall prevalence and clinical burden of dementia in their populations, as well as cost-benefit analysis of accurate diagnosis and improved health care.

The human capital contributed by the most able elders, particularly in early stages of the aging process (5570 years), is critically needed in developing countries; their experience contributes significantly to both economic productivity and family stability [80]. One way to visualize the economic costs of dementia is to consider the potential years of healthy life lost to disability (loss of function) and to premature death, through the concept of disability-adjusted life years (DALYs) [81]. Economic impact of disease also can be assessed by quality-adjusted life year (QALY), which combines duration and quality of life [82]. Since most of the dementia cases diagnosed in the developing world are of moderate to advanced severity, the price paid by society, either in DALYs or QALYs, is very high.

Cost-comparison analyses use the costs of diagnosis and of pharmacological and psychosocial interventions and other relevant costs to estimate the economic benefits of early diagnosis and early interventions [58, 83, 84]. A lack of mortality and natural history data make such calculations difficult for LMICs. Although not entirely projectable to resource-poor areas, a few general conclusions have been drawn from studies of developed countries [83-85]. First, the net benefits of early identification and intervention exceed costs, with significant savings expected. Second, the earlier the diagnosis and intervention, the greater the net benefits. Finally, caregiver interventions at earlier stages have beneficial economic effects. However, these analyses were not based on direct observations or randomized controlled trials, and no such analyses have been made for countries in the developing world.

\section{Ethical Issues Related to Diagnosis of Dementia in LMIC}

Perceptions of the nature of disease, what social scientists call social representations of disease [86], vary widely among different populations and ethnic groups, resulting in the need to adapt educational and counseling programs related to dementia [42]. Low levels of education and health literacy, poor access to health care, and the fact that many elderly subjects live within extended families pose ethical dilemmas that need to be discussed with healthcare workers before an early detection program is implemented in a resource-poor community.

The key ethical principles guiding the process of disclosure of diagnosis at early stages in the disease are beneficence, respect for the autonomy of patients, and justice for patients [87, 88]. In other words, the clinician weighs the possible benefits and harm of disclosure, and this process is related to perceptions about the ability of the patient to understand and/or retain information, the potential psychological impact, and beliefs about the efficacy of treatments [89]. Respecting the patient's autonomy is based on the notion that every individual has a right to control his/her life, as stated in Black's Alzheimer Bill of Rights [90]. The principle of justice involves hearing the voice of patients, acknowledging their vulnerabilities, giving them a chance to decide important issues while decision making is still possible, and not marginalizing them.

Unfortunately, no one has studied preferences or the impact of diagnostic disclosure in developing countries among patients, caregivers, and health professionals. It could be difficult to persuade family members that some signs and symptoms in their elderly relatives are due to a disease of the brain, and not just to the process of aging. The belief that an affected individual will become seriously dependent can have disastrous effects, including marital breakdown, suicide by the patient, and social stigma for family members [11, 91]. Attitudes toward dementia diagnoses are often based on religious or spiritual beliefs [92]. These issues are even more sensitive when a dominant gene is implicated in the dementia, possibly resulting in discrimination and/or exclusion [93]. Until research on the impact of disclosure is 
conducted in developing countries, the decision to disclose a diagnosis must depend on the probable impact on a particular patient: "If a patient is likely to benefit, he or she should be told, but if benefit is not likely, or if disclosure is instead apt to bring about an adverse reaction, the disclosure is not advised" [94].

\section{The Role of International Partnerships in Improving Diagnosis of Dementia in Resource-Poor Countries}

Partnerships between wealthy countries and LMICs (North/South partnerships) and partnerships between LMICs that have expertise in dementia and adjacent countries with no such expertise (South/South partnerships) are two strategies for improving diagnosis of dementia in resource-poor populations. However, those strategies have been implemented parsimoniously, and with few exceptions, large international funding organizations have shown little interest in partnering with LMICs to build capacity for dementia diagnosis.

Some genuine partnerships encompassing research and capacity building for delivery of services in developing countries have been extremely successful, not only in terms of scientific productivity, but also in the creation of services and dementia care options [95-97]. The research components of these partnerships have primarily been funded through governments and charitable organizations of high-income countries (North/ South partnerships). Several agencies, including the Fogarty International Center of the NIH, have developed a program called "Brain Disorders in the Developing World: Research Across the Lifespan." The program includes research and capacity building and has funded several initiatives devoted to dementia.

Only $8 \%$ of mental health projects to date involved collaboration between LMICs, as compared with $30 \%$ that involved collaboration with high-income countries [98]. Capacity building has less often been included in South/ South partnerships, which have generally been supported by a variety of sources, including individual donors and charitable organizations. In most cases, the resulting programs have been expected to be self-sustaining after the development of services. However, the potential of South/South collaborations is beginning to emerge as a significant mechanism for empowering countries in resource-poor areas. Such partnerships can provide alternative sources of financial and technical assistance [99], like the collaboration in which Indian universities have provided virtual classes for medical staff and online consultations in Africa [99]. However, funding allocated for research on mental and neurological conditions in LMICs is vastly insufficient, and lobbying of international organizations by coalitions of
LMICs might be needed to gain external support for South/South partnerships.

The main goals of international partnerships should be to accelerate diagnosis and provision of services for dementia in resource-poor regions, empowering locals through training programs that include dementia as one component. The partnerships should develop connections with the governments of LMICs and should educate the general population about dementia as a public health problem.

\section{Conclusions}

As infant and childhood mortality rates decline and life expectancies lengthen in LMICs, increases in both total population size and the proportion of elderly individuals will make dementia an increasingly severe burden on healthcare resources. Prevalence of dementia is already high in some developing countries. In Africa, India, and Bangladesh, where prevalence of dementia is currently low, rapidly increasing populations of elderly and increasing prevalence of risk factors, such as HIV-related dementia and traumatic brain injury, foretell a rise in dementia patients that is a disaster waiting to happen. Local governments are unlikely to switch their attention from ongoing problems of malnutrition and communicable disorders to an "incurable" disease like dementia. Furthermore, prevalence of many preventable chronic diseases, such as diabetes and cardiovascular disease, is increasing as LMICs adopt westernized lifestyles, further increasing pressure on limited healthcare resources.

There are currently no preventive measures or cures for dementia. Even if promising immunological approaches, gene therapy, or other strategies turn out to be safe and effective, they are likely to be expensive. Therefore, better management is the most appropriate approach for dealing with dementia in the developing world. However, it appears unlikely that funding agencies will develop initiatives to improve diagnosis of dementia in developing countries in the near future. Thus, it is vital for the international healthcare community to take action now to strengthen existing initiatives, develop North/South and South/South partnerships, and build capacity for dementia diagnosis in resourcepoor populations.

Acknowledgements The author thanks innumerable colleagues who have been involved in designing, reviewing, and executing programs for amelioration of dementia in developing countries. The progress to date would not have been possible without the help of Raj Kalaria, Martin Prince, Richard Mayeux, Yaakov Stern, Raul Arizaga, Francisco Lopera, Ricardo Nitrini, Daisy Acosta, Ciro Gaona, and Martin Medrano. Finally, thanks go to Evelyn Córdoba Villanueva for her extraordinary help with the bibliographic references. 
Disclosure The author has no conflicts of interest to disclose. G.E.M. is funded by Award Number R01AG036469 from the National Institute on Aging.

Open Access This article is distributed under the terms of the Creative Commons Attribution License which permits any use, distribution, and reproduction in any medium, provided the original author(s) and the source are credited.

\section{References}

Papers of particular interest, published recently, have been highlighted as:

- Of importance

- Of major importance

1. Sousa RM, et al. Contribution of chronic diseases to disability in elderly people in countries with low and middle incomes: A 10/66 Dementia Research Group population-based survey. Lancet. 2009;374(9704):1821-30.

2. Aisen PS. Pre-dementia Alzheimer's trials: overview. J Nutr Health Aging. 2010;14(4):294.

3. Alzheimer's Disease International, World Alzheimer's Report. 2009. Prince M, Jackson, J, editors. London: Alzheimer's Disease International; 2009.

4. Nitrini R, et al. Prevalence of dementia in Latin America: A collaborative study of population-based cohorts. Int Psychogeriatr. 2009;21(4):622-30.

5. Maestre GE. Dementia in Latin America and the Caribbean: an overlooked epidemic. Neuroepidemiology. 2008;31(4):252-3.

6. Sternberg SA, Wolfson C, Baumgarten M. Undetected dementia in community-dwelling older people: the Canadian Study of Health and Aging. J Am Geriatr Soc. 2000;48(11):1430-4.

7. - Alzheimer's Disease International, World Alzheimer Report. 2011. The benefits of early diagnosis and intervention. Prince M, Brice R, Ferri C, editors. London: Alzheimer's Disease International; 2011. Thoroughly reviews the literature on the benefits of early diagnosis, with particular emphasis on low-to-middle-income countries.

8. World Health Organization. Mental Health Gap Action Programme: scaling up care for mental, neurological and substance use disorders. Geneva: World Health Organization; 2008.

9. •- World Health Organization, Alzheimer's Disease International. Dementia: a public health priority. 2012: Geneva. p. 112. Reviews the literature on the current status and need to address the high global numbers of people with dementia with special emphasis on the situation in low-to-middle-income countries is comprenhensive.

10. Ferri CP, et al. Global prevalence of dementia: a Delphi consensus study. Lancet. 2005;366(9503):2112-7.

11. Ineichen B. The epidemiology of dementia in Africa: A review. Soc Sci Med. 2000;50(11):1673-7.

12. Acosta-Baena N, et al. Pre-dementia clinical stages in presenilin 1 E280A familial early-onset Alzheimer's disease: A retrospective cohort study. Lancet Neurol. 2011;10(3):213-20.

13. Ceaser M. Left by the lakeside. Lancet. 2004;364(9434):569-70.

14. Kaur M, Balgir PP. APOE2 and consanguinity: A risky combination for Alzheimer's disease. J Alzheimers Dis. 2005;8 (3):293-7.

15. Jorm AF, Jolley D. The incidence of dementia: A meta-analysis. Neurology. 1998;51(3):728-33.
16. U.S. Census Bureau, International Data Base. 2012. http:// www.census.gov/population/international/data/idb/information Gateway.php. Retrieved 25 March 2012.

17. Sinha RN. Make dementia a public health priority in India. Indian J Public Health. 2011;55(2):67-9.

18. Lopez OL. The growing burden of Alzheimer's disease. Am J Manag Care. 2011;17(13):S339-45.

19. Kelishadi R. Childhood overweight, obesity, and the metabolic syndrome in developing countries. Epidemiol Rev. 2007;29:62-76.

20. Boissonnet $\mathrm{C}$, et al. Educational inequalities in obesity, abdominal obesity, and metabolic syndrome in seven Latin American cities: The CARMELA Study. Eur J Cardiovasc Prev Rehabil. 2011;18 (4):550-6.

21. Subramanian SV, et al. Weight of nations: a socioeconomic analysis of women in low- to middle-income countries. Am J Clin Nutr. 2011;93(2):413-21.

22. Popkin BM. The shift in stages of the nutrition transition in the developing world differs from past experiences! Public Health Nutr. 2002;5(1A):205-14.

23. McKhann G, et al. Clinical diagnosis of Alzheimer's disease: report of the NINCDS-ADRDA Work Group under the auspices of Department of Health and Human Services Task Force on Alzheimer's Disease. Neurology. 1984;34(7):939-44.

24. McKeith IG, et al. Consensus guidelines for the clinical and pathologic diagnosis of dementia with Lewy bodies (DLB): report of the consortium on DLB international workshop. Neurology. 1996;47(5):1113-24.

25. Zerr I, et al. Updated clinical diagnostic criteria for sporadic Creutzfeldt-Jakob disease. Brain. 2009;132(Pt 10):2659-68.

26. Sperling RA, et al. Toward defining the preclinical stages of Alzheimer's disease: recommendations from the National Institute on Aging-Alzheimer's Association workgroups on diagnostic guidelines for Alzheimer's disease. Alzheimers Dement. 2011;7 (3):280-92.

27. McKhann GM, et al. The diagnosis of dementia due to Alzheimer's disease: recommendations from the National Institute on AgingAlzheimer's Association workgroups on diagnostic guidelines for Alzheimer's disease. Alzheimers Dement. 2011;7(3):263-9.

28. Jack $\mathrm{Jr} \mathrm{CR}$, et al. Introduction to the recommendations from the National Institute on Aging-Alzheimer's Association workgroups on diagnostic guidelines for Alzheimer's disease. Alzheimers Dement. 2011;7(3):257-62.

29. Mayeux R, Stern Y, Spanton S. Heterogeneity in dementia of the Alzheimer type: evidence of subgroups. Neurology. 1985;35 (4):453-61.

30. Kaye JA. Diagnostic challenges in dementia. Neurology. 1998;51 (1):S45-52. discussion S65-7.

31. Baiyewu O. Dementia in Nigeria. Archives of Ibadan Medicine. 2003;4(1):21-2.

32. Muela SH, Mushi AK, Ribera JM. The paradox of the cost and affordability of traditional and government health services in Tanzania. Health Policy Plan. 2000;15(3):296-302.

33. Romero-Salazar A, Roque-Rujano R, Maestre GE. Knowlwdge, beliefs and report of the practice of general practicioners in primary attention in Maracaibo, Venezuela about dementia. Investigacion en Salud. 2007;9(1):10-5.

34. Dias A, Patel V. Closing the treatment gap for dementia in India. Indian J Psychiatry. 2009;51 Suppl 1:S93-7.

35. Maestre GE, Rojas-Ruiz OM, Biomedicalization of cognitive deficits: Lessons from the Maracaibo Aging Study and implications for developing countries. Alzheimer's \& Dementia, 2009. 5(4): p. P91.

36. Boggatz T, Dassen T. Ageing, care dependency, and care for older people in Egypt: a review of the literature. J Clin Nurs. 2005;14 (8B):56-63

37. Chu LW, Chi I. Nursing homes in China. J Am Med Dir Assoc. 2008;9(4):237-43. 
38. Ogunniyi A, et al. Caring for individuals with dementia: The Nigerian experience. West Afr J Med. 2005;24(3):259-62.

39. Perry B, Gesler W. Physical access to primary health care in Andean Bolivia. Soc Sci Med. 2000;50(9):1177-88.

40. Patel V, Prince M. Ageing and mental health in a developing country: Who cares? Qualitative studies from Goa, India. Psychol Med. 2001;31(1):29-38.

41. Pentzek M, et al. Apart from nihilism and stigma: What influences general practitioners' accuracy in identifying incident dementia? Am J Geriatr Psychiatry. 2009;17(11):965-75.

42. Van Gorp B, Vercruysse T. Frames and counter-frames giving meaning to dementia: A framing analysis of media content. Soc Sci Med. 2012;74(8):1274-81.

43. Fernando SM, Deane FP, McLeod HJ. Sri Lankan doctors' and medical undergraduates' attitudes towards mental illness. Soc Psychiatry Psychiatr Epidemiol. 2010;45(7):733-9.

44. Finkel SI, et al. Behavioral and psychological signs and symptoms of dementia: A consensus statement on current knowledge and implications for research and treatment. Int Psychogeriatr. 1996;8 Suppl 3:497-500.

45. Guerchet M, et al. Factors associated with dementia among elderly people living in two cities in Central Africa: The EDAC multicenter study. Journal of Alzheimer's Disease. 2012;29(1):15-24.

46. Kwok JB. Role of epigenetics in Alzheimer's and Parkinson's disease. Epigenomics. 2010;2(5):671-82.

47. Olarte L, et al. Apolipoprotein E epsilon4 and age at onset of sporadic and familial Alzheimer disease in Caribbean Hispanics. Arch Neurol. 2006;63(11):1586-90.

48. Sinforiani E, et al. The effects of alcohol on cognition in the elderly: From protection to neurodegeneration. Funct Neurol. 2011;26(2):103-6.

49. Chaves, M.L., et al., Predictors of the progression of dementia severity in brazilian patients with Alzheimer's disease and vascular dementia. Int J Alzheimers Dis, 2010. 2010

50. - Mayeux R, et al. Operationalizing diagnostic criteria for Alzheimer's disease and other age-related cognitive impairment-Part 1. Alzheimers Dement. 2011;7(1):15-34. Reviews differences in case assessment, methodological biases, cultural and educational influences on test performance, inclusion of special populations, such as underrepresented minorities and the oldest old, and detection of the earliest symptomatic stages of underlying Alzheimer's disease.

51. Mathew R, Mathuranath PS. Issues in evaluation of cognition in the elderly in developing countries. Ann Indian Acad Neurol. 2008;11(2):82-8.

52. Gomez F, Curcio CL, Duque G. Health care for older persons in Colombia: a country profile. J Am Geriatr Soc. 2009;57(9):1692-6.

53. Heng MB, Key PJ. Cambodian health in transition. BMJ. 1995;311 (7002):435-7.

54. Saraceno B, et al. Barriers to improvement of mental health services in low-income and middle-income countries. Lancet. 2007;370(9593):1164-74.

55. Petersen I, Lund C, Stein DJ. Optimizing mental health services in low-income and middle-income countries. Curr Opin Psychiatry. 2011;24(4):318-23.

56. Devanand DP, et al. Predictive utility of apolipoprotein E genotype for Alzheimer disease in outpatients with mild cognitive impairment. Arch Neurol. 2005;62(6):975-80.

57. Ashford JW, et al. Should older adults be screened for dementia? It is important to screen for evidence of dementia! Alzheimers Dement. 2007;3(2):75-80.

58. Cappell J, et al. The pharmacoeconomics of cognitive enhancers in moderate to severe Alzheimer's disease. CNS Drugs. 2010;24 (11):909-27.

59. Kalula SZ, et al. Profile and management of patients at a memory clinic. S Afr Med J. 2010;100(7):449-51.
60. Alladi S, et al. Subtypes of dementia: a study from a memory clinic in India. Dement Geriatr Cogn Disord. 2011;32(1):32-8.

61. Maria Benabdeljlil, F.B., M. Rahmani, F. Benbelaïd, M. Bennani, S. Aïdi, M. El Alaoui Faris. Dementia and Alzheimer's disease. Experience of the Memory Clinic of Rabat. in XXth World COngress of Neurology. 2011. Marrakesh, Morocco.

62. Jolley D, Moniz-Cook E. Memory clinics in context. Indian $\mathrm{J}$ Psychiatry. 2009;51 Suppl 1:S70-6.

63. Saxena $\mathrm{S}$, et al. Resources for mental health: scarcity, inequity, and inefficiency. Lancet. 2007;370(9590):878-89.

64. Kakuma R, et al. Human resources for mental health care: current situation and strategies for action. Lancet. 2011;378(9803):1654 63.

65. Araya M, Mussie M, Jacobson L. Decentralized psychiatric nursing service in Ethiopia-a model for low income countries. Ethiop Med J. 2009;47(1):61-4.

66. Mateen FJ. International issues: neurology in Bangladesh. Neurology. 2010;74(2):e5-8.

67. Shi FD, Jia JP. Neurology and neurologic practice in China. Neurology. 2011;77(22):1986-92.

68. Bruckner TA, et al. The mental health workforce gap in low- and middle-income countries: a needs-based approach. Bull World Health Organ. 2011;89(3):184-94.

69. Adair J, Kagitcibasi C. Development of Psychology in Developing COuntries: Factors Facilitatng and Impeding its Progress. International Journal of Psychology. 1995;30 (6):633-41.

70. World Health Organization, The World Health Report 2001, in Mental Health: new understanding, new hope. 2001: Geneva.

71. Prince MJ, et al. Packages of care for dementia in low- and middleincome countries. PLoS Med. 2009;6(11):e1000176.

72. Lee L, Kasperski MJ, Weston WW. Building capacity for dementia care: Training program to develop primary care memory clinics. Can Fam Physician. 2011;57(7):e249-52.

73. Perry M, et al. Can an EASYcare based dementia training programme improve diagnostic assessment and management of dementia by general practitioners and primary care nurses? The design of a randomised controlled trial BMC Health Serv Res. 2008;8:71.

74. Downs M, et al. Effectiveness of educational interventions in improving detection and management of dementia in primary care: cluster randomised controlled study. BMJ. 2006;332 (7543):692-6.

75. Hobday JV, et al. Feasibility of Internet training for care staff of residents with dementia: The CARES program. J Gerontol Nurs. 2010;36(4):13-21.

76. Vollmar HC, et al. Knowledge translation on dementia: a cluster randomized trial to compare a blended learning approach with a "classical" advanced training in GP quality circles. BMC Health Serv Res. 2007;7:92.

77. Ogbaini-Emovon E. Continuing medical education: Closing the gap between medical research and practice. Benin Journal of Postgraduate Medicine. 2009;11:43-9. Supplemental December.

78. Morris L, Taitsman JK. The agenda for continuing medical education-limiting industry's influence. N Engl J Med. 2009;361 (25):2478-82.

79. Allegri RF, et al. Economic impact of dementia in developing countries: An evaluation of costs of Alzheimer-type dementia in Argentina. Int Psychogeriatr. 2007;19(4):705-18.

80. UN Department of Economic and Social Affairs. Regional Dimensions of the Ageing Situation, New York: United Nations; 2008.

81. Murray C, Lopez AD. The Global Burden of Disease: A comprehensive assessment of mortality and disability from diseases, injuries and risk factors in 1990 and projected to 2020. Cambridge, MA: Harvard Unievrsity Press; 1996. 
82. Zeckhauser RSD. Where now for saving lives? Law and Contemporary Problems. 1976;40:5-45.

83. Weimer DL, Sager MA. Early identification and treatment of Alzheimer's disease: social and fiscal outcomes. Alzheimers Dement. 2009;5(3):215-26.

84. Banerjee S, Wittenberg R. Clinical and cost effectiveness of services for early diagnosis and intervention in dementia. Int J Geriatr Psychiatry. 2009;24(7):748-54.

85. Getsios D, et al. An economic evaluation of early assessment for Alzheimer's disease in the United Kingdom. Alzheimers Dement. 2012;8(1):22-30.

86. Moscovici S. The phenomenon of social representations. In: Farr RM, Moscovici S, editors. Social Representations. Cambridge: Cambridge University Press; 1986.

87. Marzanski M. On telling the truth to patients with dementia. West J Med. 2000;173(5):318-23.

88. Post SG, Whitehouse PJ. Fairhill guidelines on ethics of the care of people with Alzheimer's disease: a clinical summary. Center for Biomedical Ethics, Case Western Reserve University and the Alzheimer's Association. J Am Geriatr Soc. 1995;43(12):1423-9.

89. Bamford C, et al. Disclosing a diagnosis of dementia: A systematic review. Int J Geriatr Psychiatry. 2004;19(2):151-69.

90. Black JS. Telling the truth: Should persons with Alzheimer's disease be told their diagnosis? Alzheimer's Disease International Global Perspective. 1995;6:10-1.

91. Cheng ST, Lam LC, Chan LC, Law AC, Fung AW, Chan WC, Tam $\mathrm{CW}$, Chan WM. The effects of exposure to scenarios about dementia on stigma and attitudes toward dementia care in a Chinese community. International Psychogeriatrics/IPA. 2011;23:1433-41.

92. Ng TP, Niti M, Chiam PC, Kua EH. Religion, health beliefs and the use of mental health services by the elderly. Aging and Mental Health. 2011;15(2):143-9.

93. Williams JK, et al. In their own words: reports of stigma and genetic discrimination by people at risk for Huntington disease in the International RESPOND-HD study. American Journal of Medical Genetics Part B: Neuropsychiatric Genetics. 2010;153B (6):1150-9.

94. Carpenter B, Dave J. Disclosing a dementia diagnosis: a review of opinion and practice, and a proposed research agenda. Gerontologist. 2004;44(2):149-58.

95. Hendrie HC, et al. Alzheimer's disease, genes, and environment: the value of international studies. Can J Psychiatry. 2004;49(2):92-9.

96. Prince MJ. The 10/66 dementia research group - 10 years on. Indian J Psychiatry. 2009;51 Suppl 1:S8-S15.

97. Hendrie HC. Lessons learned from international comparative crosscultural studies on dementia. Am J Geriatr Psychiatry. 2006;14(6):480-8.

98. Razzouk D, et al. Scarcity and inequity of mental health research resources in low-and-middle income countries: a global survey. Health Policy. 2010;94(3):211-20.

99. Sridhar D. Seven challenges in international development assistance for health and ways forward. J Law Med Ethics. 2010;38 (3):459-69. 